\title{
Psychology Software Tool (PST) For Specific Language Impairment Person
}

\author{
Y.H. Ch'ng', Mohd Azam Osman*², H.Y. Jong ${ }^{3}$ \\ ${ }^{1, * 2}$ School of Computer Sciences, Universiti Sains Malaysia, 11800 USM Pulau Pinang, Malaysia \\ ${ }^{3}$ School of Humanities, Universiti Sains Malaysia, 11800 USM Pulau Pinang, Penang, Malaysia \\ 1'chng_yan_huan@student.usm.my, ${ }^{2 *}$ azam@usm.my, ${ }^{3}$ jonghuiying@usm.my
}

Article History: Received: 10 November 2020; Revised: 12 January 2021; Accepted: 27 January 2021; Published online: 05 April 2021

Abstract: Specific Language Impairment (SLI) is a disorder categorized by the inability of an individual to master spoken
and written language, despite the absence of any apparent handicapping conditions. Many tools have been developed to
diagnose or treat SLI, but these tools are largely made up of standalone components which must be utilized separately to
obtain sufficient data for the diagnosis and treatment of SLI. Furthermore, some of these components are either very
expensive or not widely available. As a result, therapists have faced many inconveniences when trying to treat individuals
suffering from SLI. Hence, the development of an integrated, cost-effective Psychology Software Tool (PST) to diagnose and
treat SLI is being developed as a solution to counter the inconveniences currently faced by SLI therapists. As a tool by SLI
therapists to diagnose and treat SLI within children, the Psychology Software Tool offers standardized questionnaires with a
special method to diagnose SLI will be presented in both text and audio, while a webcam will be used to deduce the amount
of focus which is given by the patient to comprehend a particular element displayed on the screen. The diagnosis shall take
into account the accuracy of answers provided within the questionnaires, the time taken to provide those answers and the
aforementioned degree of focus. In terms of SLI treatment, the tool will also provide music therapy for SLI patients to work
towards better speech production and comprehension. In short, PST will simplify and enhance the process of diagnosing and
treating SLI patients. Keywords: Specific Language Impairment, Diagnosis, Music therapy

\section{Introduction}

Specific Language Impairment (SLI), also known as Developmental Language Disorder (DLD) is a disorder wherein an individual has an apparent delay in language development although they do not appear to be inhibited physically or intellectually. An individual suffering from SLI may experience difficulties producing words verbally, learning new words and making conversations. More prominent symptoms also include long delays in order to comprehend a written or spoken sentence. Rice (2013) stated that SLI is especially common among children and adolescents, such that approximately 7 to 8 percent of children in kindergarten are affected, and the problems introduced by SLI would persist into adulthood. The exact cause of SLI is unknown, but it is suggested that the disorder is passed down genetically. Needless to say, if SLI is not diagnosed and treated correctly, it will negatively affect a child's performance in school.

Traditionally, the diagnosis and treatment of SLI is carried out manually by speech-language pathologists and therapists. Standardized tests to aid diagnosis of SLI were then developed and used in schools to screen for cases of language impairments. As the integration of technology began to take place in the medical field, more advanced tools like eye trackers and electroencephalogram (EEG) bio-sensors became accessible to therapists, which allow them to determine cases of SLI at higher ease and accuracy by acquiring data on their patient's attention and point of focus within a diagnosis. More studies with respect to the automation of various SLI diagnosis and treatment procedures began to surface, as in Gong et al. (2016), Gerosa et al. (2007), Richards et al. (2017), Dykstra et al. (2012), Hussain et al., (2017) and Greenwood et al. (2018). Despite the many advancements and research made to better understand the nature of SLI, and better ways to diagnose and treat children with it, both diagnosis and treatment of SLI remain daunting tasks for two main reasons. First of all, the aforementioned advanced tools to diagnose SLI, such as eye trackers and EEG bio-sensors are expensive and not readily available to all therapists and researchers. Farnsworth (2019) discusses how mid-range eye trackers can cost up to $\$ 10,000$, and while high-end eye trackers mainly used for research purposes cost even more than that, low-end eye trackers which are not recommended for research use still cost as much as $\$ 1,000$. EGG bio-sensors which evaluate electrical activity in the brain go up to $\$ 25,000$, and are mainly available only in professional health facilities such as hospitals. The second problem involves a lack of integration among different tools used to diagnose and treat SLI. Records of scores obtained from standard questionnaires displayed in one program, are then fed to a different program which builds a Receiver Operating Characteristic (ROC) curve for further interpretation. If an eye tracker is used, the data may be collected separately in another different process, all of which are only compiled together at the end of the diagnosis, by hand. As if that isn't enough, the treatment process for SLI also requires different tools and programs. In order to complete a SLI diagnosis alone, the pathologist or therapist in charge has to learn how to utilize different tools, many of which are not within their 
field of expertise. This makes the already complex task of diagnosing and treating SLI, unnecessarily difficult and even chaotic. As a result, the current process of dealing with SLI is inefficient took to provide those answers and their degree of focus on certain elements displayed on the screen.

This paper presents an all-in-one Psychology Software Tool (PST) which can be used by a therapists or a speech-language pathologist to diagnose and treat SLI, without the use of costly equipment which are not widely available in our everyday lives. The Psychology Software Tool is designed as a single web application capable of delivering effective SLI diagnosis and treatment, in which dependencies on non-integrated tools and costly equipment are eliminated.

\section{Materials and Methods}

The Psychology Software Tool is an integrated package capable of delivering both SLI diagnosis and SLI treatment. It is a single web application with fixed methods selected for SLI diagnosis and treatment, designed for the use of children suffering from SLI, under the supervision of speech language pathologists or therapists. There are many techniques used to diagnose and evaluate the severity of SLI of a patient. These techniques may differ based on the age of the patient, particular expertise of the speech language pathologist or therapists, and the prevalence of certain symptoms exhibited by the patient. Likewise, there are many techniques to treat SLI. The Psychology Software Tool focuses on implementing binary picture matching task for SLI diagnosis and rhythm beat music therapy for SLI treatment. Fig. 1 shows a summarized flow of the procedures of diagnosing SLI. In terms of SLI diagnosis, the Psychology Software Tool adheres to the standards of comprehension assessment procedures via a binary sentence-picture matching task. Users undergoing diagnosis will listen to a semantically reversible sentence, and attempt to match the sentence to one of two pictures which is most accurately described by the sentence. The steps of an SLI diagnosis provided by the Psychology Software Tool are listed below.

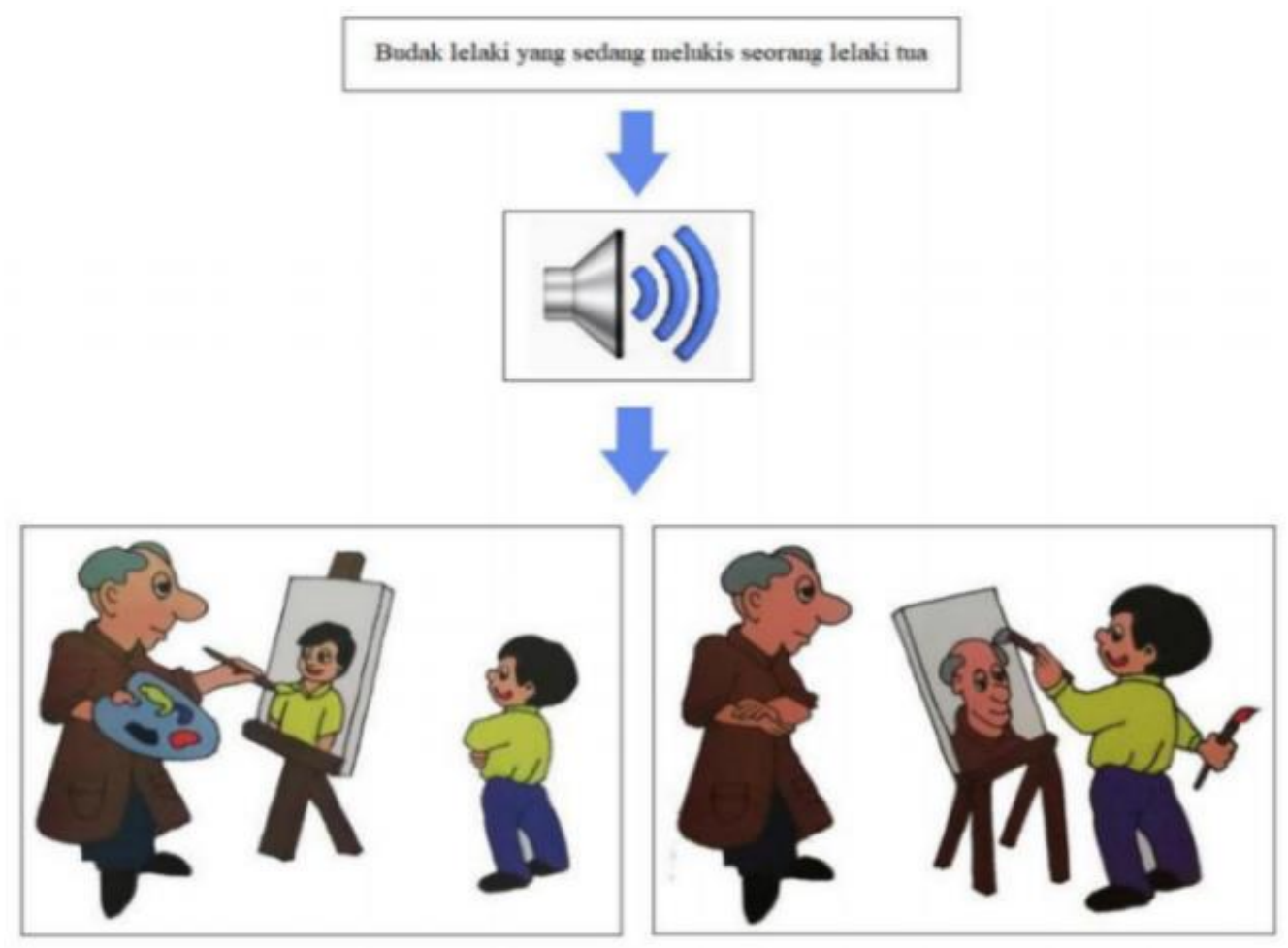

Figure 1. SLI Diagnosis procedures

Upon starting a diagnosis session, a black, blank screen is displayed.

After $1000 \mathrm{~ms}$, a sentence is displayed for $5000 \mathrm{~ms}$.

Audio of the sentence is then provided within 7000ms.

A black, blank screen is displayed again for 500ms.

Two pictures are shown; the user selects one which matches the sentence

Throughout the listed steps, the user's webcam will be accessed through their web browser to infer the point of focus of their gaze. Subsequently, a heat map will be generated to help the therapist or pathologist to further 
assess the patient's condition. The complete diagnosis shall take into account multiple parameters such as the accuracy of answers provided by the patient, the time they took to provide those answers and their degree of focus on certain elements displayed on the screen.

In terms of SLI treatment, the Psychology Software Tool adopts music therapy as a medical treatment to improve on the symptoms of SLI. Since the left hemisphere of the brain is responsible for language and speech, having SLI implies that parts of the left hemisphere of the brain is impaired. Music, on the other hand, has been regarded as a right-brain activity due to its reliance on creativity. However, music therapy has also been proven to improve motor skills and hand-eye coordination, both of which is central to speech and hearing development, as discussed in Iverson (2010). Fig. 2 depicts how SLI treatment via music therapy is being implemented in the Psychology Software Tool.
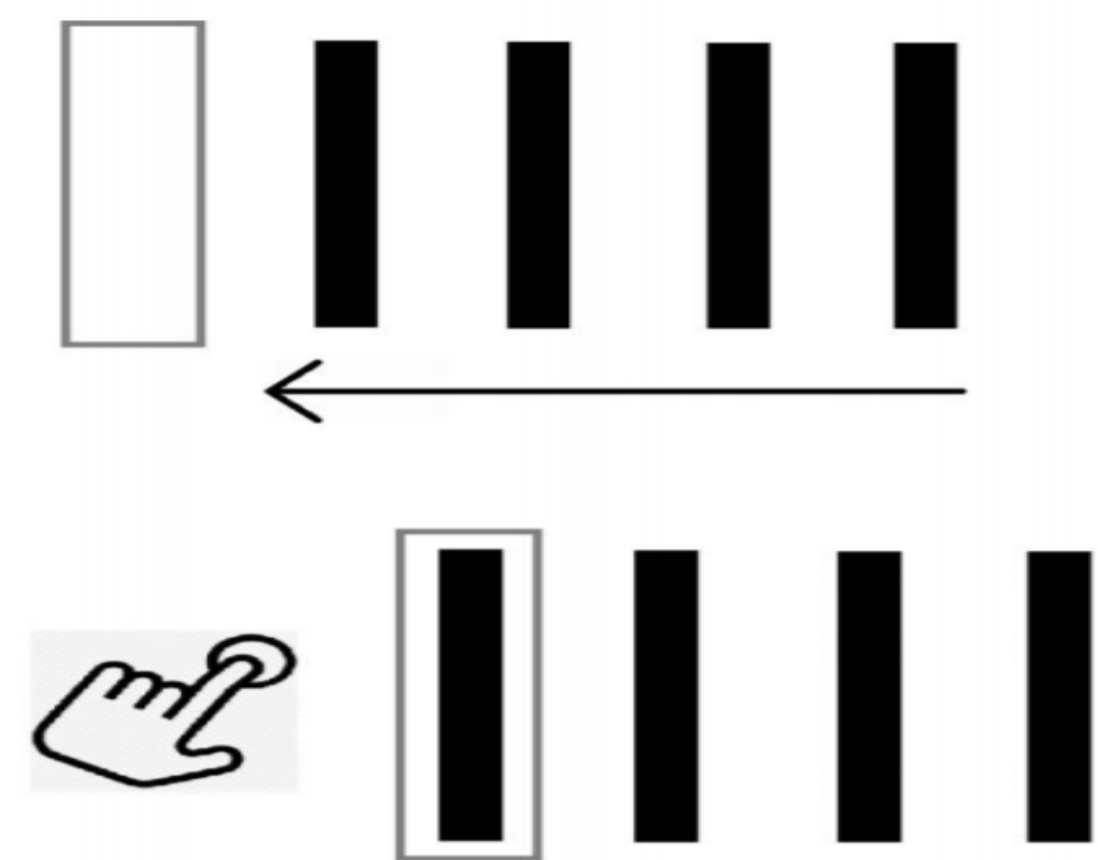

Figure 2. Music Therapy Visualization

Traditionally, patients undergoing music therapy were tasked to clap to the rhythm of music. Music therapy is about training motor skills and hand-eye coordination, so a stable and rhythmic tune would be deployed and users undergoing the therapy would be tasked to "hit to the beat" while being assisted by visuals such as bars travelling into a hit box on the screen.

In terms of designing the architecture of the system, crucial elements of the system has been taken into consideration and the system architecture diagram of the Psychology Software Tool has been designed, as shown in Fig. 3. The Psychology Software Tool will be utilized by two types of users, namely the therapist and the patient. The first user group plays a supervisory role overseeing the diagnosis process of a potential SLI patient. This user can be a SLI therapist, a researcher, or a speech-language pathologist. The second user group involves the potential SLI patients who would undergo diagnosis and treatment for SLI. A webcam and computer with access to the internet is required to access the Psychology Software Tool. The tool will accept and evaluate questionnaire answers, webcam data which helps to deduce the amount of focus given by a patient to comprehend certain on-screen elements, and other parameters such as the time taken to provide the answers. Based on these parameters, the tool will then return information pertaining to the result of the SLI diagnosis. 


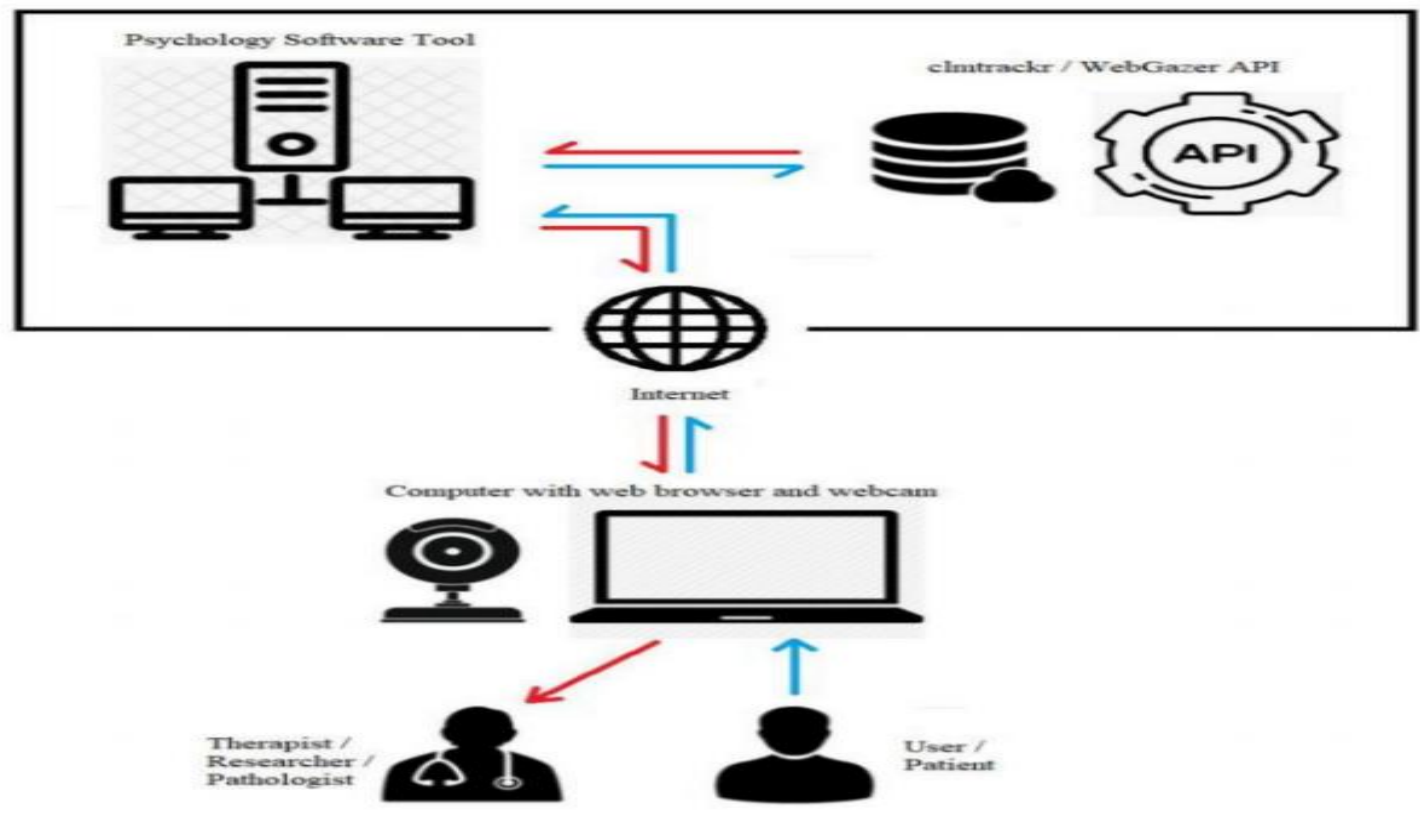

Figure 3. Architecture Diagram of Psychology Software Tool

Prior to presenting the diagnosis results to SLI therapists and pathologists, the Psychology Software Tool will communicate with third-party libraries, such as WebGazer.js. As documented in Papoutsaki et al. (2016), WebGazer.js is capable of deducing facial landmarks and thereby obtaining user gaze coordinates from the webcam footage. Music therapy will be provided by the Psychology Software Tool as long as the user has a working internet connection and is capable of loading the web application

\section{Results and Discussion}

As of the time of writing, a prototype of the Psychology Software Tool is being put to use and tested. Although formal evaluation has yet to commence, preliminary analysis of the prototype has proven that the tool suffices as a feasible replacement to certain hardware-based eye trackers. By leveraging third-party libraries such as heatmap.js, the Psychology Software Tool is capable of producing high-quality heat maps based on captured gaze data, which has been found to be sufficiently accurate under proper lighting conditions. Fig. 4 shows the heat map which is generated as part of the SLI diagnosis results.

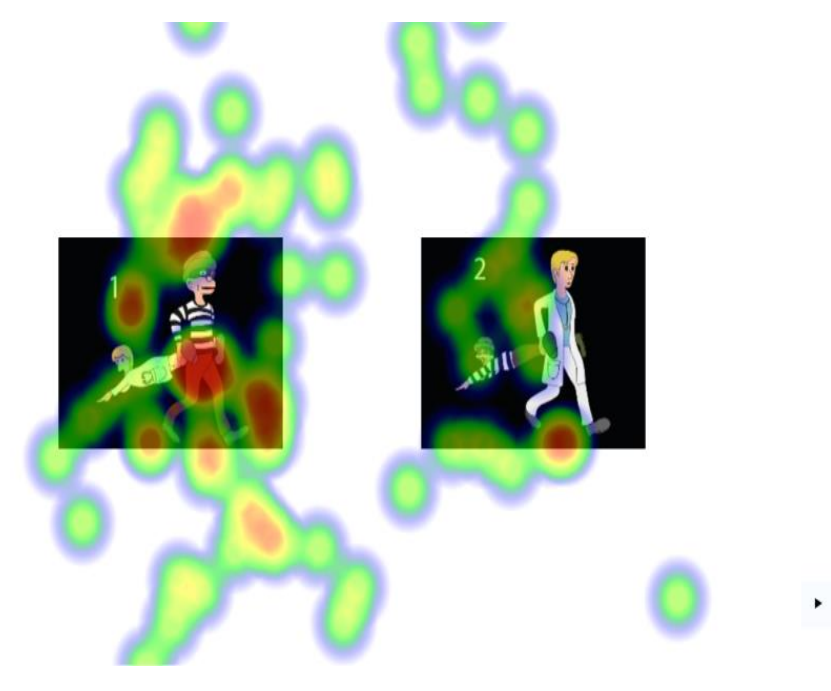

Figure 4. SLI Diagnosis - Gaze tracking heat map

Apart from heat maps, the Psychology Software Tool also produces a report on the analysis done onto the resulting score of the binary image matching task. The calculations and formula used are in accordance to the 
methodologies introduced by Crawford et al. (2002). Fig. 5 shows an example of the generated SLI diagnosis report.

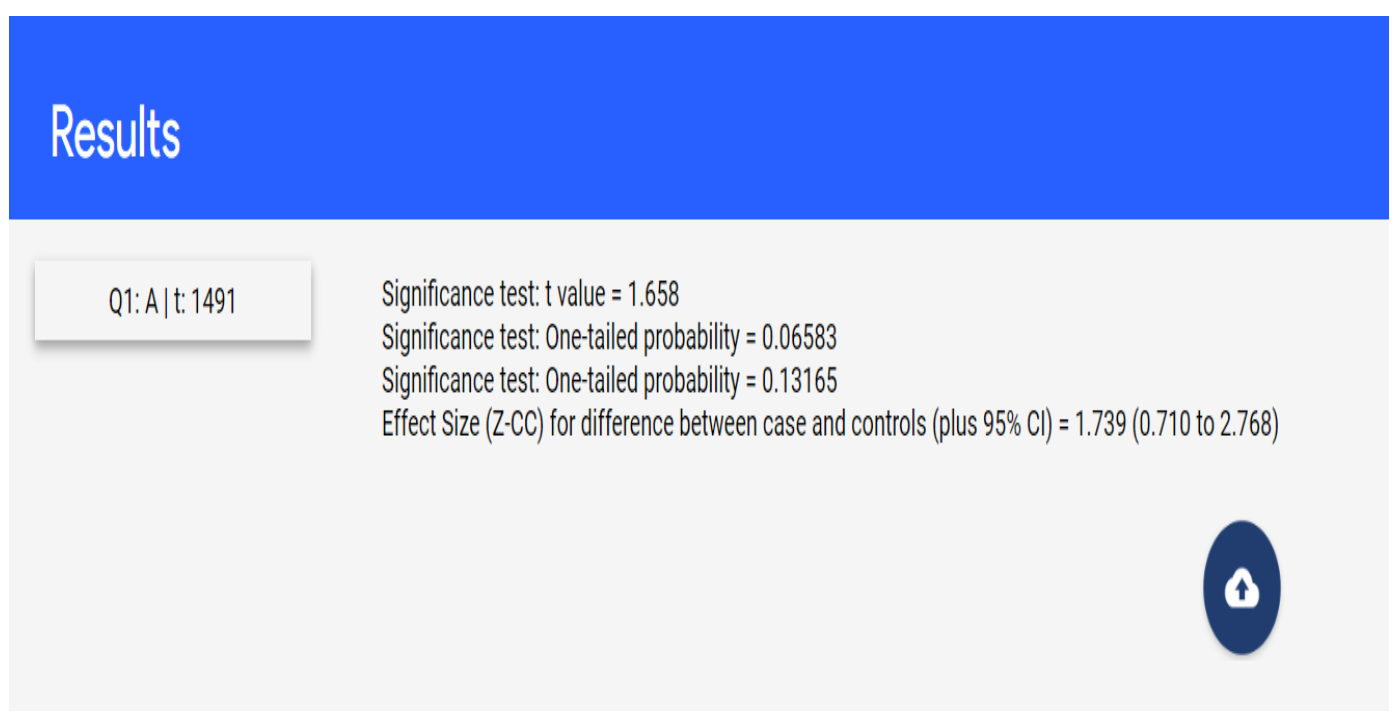

Figure 5. SLI Diagnosis - Result Analysis Report

\section{Conclusion}

For many years, people have wondered why certain children do not seem to follow instructions or understand what they have read. There are children who seem to have forgotten all the words by their next lesson in school. This can be particularly puzzling in a child who has clear talents in other areas, such as music. SLI is not known as the "hidden disorder" for no reason, and one of the main reasons behind the lack of awareness of SLI is its difficulty to be spotted and diagnosed. The Psychology Software Tool is currently being prototyped - There are plenty of aspects which need to be considered in order for a complete system design to be constructed comprehensively before production takes place to deliver the features and functionality which are required. Of course, once these aspects are taken care of, the complete Psychology Software Tool will be produced, and distributed to interested SLI therapists and pathologists. Following the clear requirements that has been elicited for the development of this tools, we adapt an optimistic outlook that the Psychology Software Tool, which not only provides computer-aided SLI diagnosis, but also music therapy, would greatly help to reduce the problems and inconveniences faced by SLI therapists when trying to diagnose and provide treatment to children.

\section{Acknowledgements}

We would like to express our deepest appreciations to all staff of both the School of Computer Sciences and the School of Humanities, USM. We gratefully acknowledge all the participants for their commitment and cooperation in the data collection process of our research. This study was supported by the short-term grant (304/PHUMANITI/6315349) from Universiti Sains Malaysia.

\section{References}

1. Rice, M. L. (2013). Language growth and genetics of specific language impairment. International Journal of Speech-Language Pathology, 15(3), 223-233. doi:10.3109/17549507.2013.783113

2. Farnsworth, B. (2019). Eye Tracker Prices: An Overview of 20+ Eye Trackers. iMotions. https://imotions.com/blog/eye-tracker-prices/

3. Iverson, J. M. (2010). Developing language in a developing body: the relationship between motor development and language development. Journal of Child Language, 37(02), 229. doi:10.1017/s0305000909990432

4. Gong, J.J., Gong, M., Levy-Lambert, D., Green, J.R., Hogan, T.P., Guttag, J.V. (2016) Towards an Automated Screening Tool for Developmental Speech and Language Impairments. Proc. Interspeech 2016, 112-116. doi:10.21437/Interspeech.2016-549

5. Weismer, S. E. (2013). Developmental Language Disorders: Challenges and Implications of Cross-Group Comparisons. Folia Phoniatrica et Logopaedica, 65(2), 68-77. doi:10.1159/000353896 
6. Gerosa, M., Giuliani, D., \& Brugnara, F. (2007). Acoustic variability and automatic recognition of children's speech. Speech 860. doi:10.1016/j.specom.2007.01.002

7. Hagen, A., Pellom, B., \& Hacioglu, K. (2009). Generating synthetic children's acoustic models from adult models. Proceedings of Human Language Technologies: The 2009 Annual Conference of the North American Chapter of the Association for Computational Linguistics, Companion Volume: Short Papers on - NAACL '09. doi:10.3115/1620853.1620877

8. Hussain, A., Mkpojiogu, E.O.C., Nawi, M.N.M. (2017). Capturing customer satisfaction and dissatisfaction in software requirements elicitation for features in proposed software systems. Journal of Engineering and Applied Sciences, 12 (21), pp. 5590-5597.

9. Dykstra, J. R., Sabatos-DeVito, M. G., Irvin, D. W., Boyd, B. A., Hume, K. A., \& Odom, S. L. (2012). Using the Language Environment Analysis (LENA) system in preschool classrooms with children with autism spectrum disorders. Autism, 17(5), 582-594. doi:10.1177/1362361312446206

10. Greenwood, C. R., Schnitz, A. G., Irvin, D., Tsai, S. F., \& Carta, J. J. (2018). Automated Language Environment Analysis: A Research Synthesis. American Journal of Speech-Language Pathology, 27(2), 853-867. doi:10.1044/2017_ajslp-17-0033

11. Richards, J. A., Xu, D., Gilkerson, J., Yapanel, U., Gray, S., \& Paul, T. (2017). Automated Assessment of Child Vocalization Development Using LENA. Journal of Speech, Language, and Hearing Research, 60(7), 2047-2063. doi:10.1044/2017_jslhr-1-16-0157

12. Paoutsaki, A., Sangkloy, P., Laskey, J., Daskalova, N., Huang, J., \& Hays, J. (2016). WebGazer: Scalable Webcam Eye Tracking Using User Interactions, Proceedings of the 25th International Joint Conference on Artificial Intelligence (IJCAI), pp 3839-3845.

13. Hosozawa, M., Tanaka, K., Shimizu, T., Nakano, T., \& Kitazawa, S. (2012). How Children With Specific Language Impairment View Social Situations: An Eye Tracking Study. PEDIATRICS, 129(6), e1453-e1460. doi:10.1542/peds.2011-2278

14. Crawford, J. R., \& Garthwaite, P. H. (2002). Investigation of the single case in neuropsychology: confidence limits on the abnormality of test scores and test score differences. Neuropsychologia, 40(8), 1196-1208. doi:10.1016/s0028-3932(01)00224-x 